\title{
Using the Fluorescent Dye, Rhodamine B, to Study Mating Competitiveness in Male Aedes aegypti Mosquitoes
}

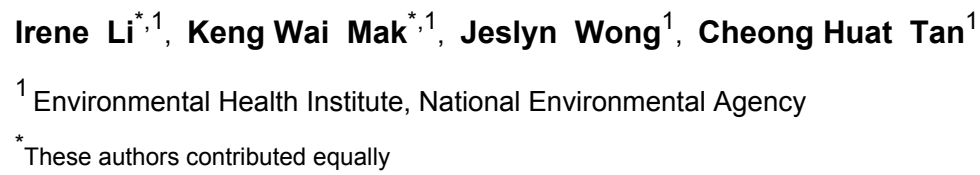

\section{Corresponding Author}

Cheong Huat Tan

tan_cheong_huat@nea.gov.sg

\section{Citation}

Li, I., Mak, K.W., Wong, J.,

Tan, C.H. Using the Fluorescent

Dye, Rhodamine B, to Study Mating Competitiveness in Male Aedes aegypti Mosquitoes. J. Vis. Exp. (171), e62432, doi:10.3791/62432 (2021).

\section{Date Published}

May 7, 2021

DOI

$10.3791 / 62432$

URL

jove.com/video/62432

\section{Abstract}

The success of sterile or incompatible insect technique-based population suppression programs depends on the ability of released males to compete for wild-type females and induce sterility in the target population. Hence, laboratory assessment of male mating competitiveness is essential for evaluating the release strain's fitness before field release. Conventionally, such an assay is performed by determining the proportion of viable eggs produced by the females after being simultaneously exposed to two sets of males (wild-type and release strains) for copulation. However, this process is time-consuming and laborious due to the need to first blood-feed the females for egg production and then hatch and enumerate the hatched eggs to determine egg viability.

Moreover, this method cannot discern the degree of competitiveness between two sterile or Wolbachia-infected mosquito lines as wild-type female mosquitoes will only produce non-viable eggs upon mating with both. To circumvent these limitations, this paper describes a more direct method of measuring male mosquito mating competitiveness in laboratory settings using the fluorescent dye, rhodamine $B(R h B)$, which can be used to mark males by feeding them in sucrose solution containing $\mathrm{RhB}$. After the mating assay, the presence of fluorescing sperms in the spermathecae of a female can be used to determine her mating partner. This method is costeffective, reduces the experimental time by $90 \%$ and allows comparison of mating fitness between two sterile or Wolbachia-infected lines.

\section{Introduction}

The rearing and release of sterile or incompatible males for suppression of Aedes mosquito populations is currently being evaluated in the field as a novel tool to prevent outbreaks of dengue and other Aedes-borne disease ${ }^{1}$. Male- 
release suppression strategies that are currently in field trials include the use of the genetic method ${ }^{2}$, irradiation (Sterile Insect Technique, SIT) ${ }^{3}$, endosymbiotic bacteria Wolbachia (Insect Incompatible Technique, IIT) ${ }^{4}$, or a combination of the latter two techniques ${ }^{5,6}$. The success of these approaches is largely dependent on the released males' ability to outcompete wild-type males and seek females to secure copulation. Otherwise, sterility cannot be induced in the target population.

In a classical SIT program, for example, male mating fitness may be affected by factors such as irradiation dose ${ }^{7,8,9}$, mass rearing protocol and the extent of inbreeding in the colony $10,11,12,13,14$. Furthermore, studies on the mating competitiveness may provide important knowledge on mosquito mating behavior which could be used to inform vector control strategies.

In SIT and IIT, the mating competitiveness of male mosquitoes is typically assessed by allowing both wild-type and release strain to compete for wild-type females in a cage $8,11,15,16$. Females are then blood-fed and their eggs hatched to determine viability. Females that lay nonviable eggs or eggs with low hatch rate are assumed to have mated with release strain males, while females that produce viable eggs are assumed to have mated with wildtype males. Mating competitiveness is then calculated with the Fried Index ${ }^{17}$. Unfortunately, this method is resourceintensive and time-consuming, and the overall Fried Index can be influenced by external confounding factors affecting egg viability such as poor egg handling and over-desiccation can result in a low hatch rate in the compatibility cross that may then lead to an artificially low Fried Index.

Moreover, this method does not allow for the direct comparison of mating competitiveness between Aedes mosquitoes that are infected with different strains of Wolbachia or that are exposed to different doses of irradiation. Hence, a more direct method is required to address these challenges. Recent studies ${ }^{18,19}$ have demonstrated the effectiveness of using the fluorescent dye, RhB, to mark male mosquitoes' seminal fluid. Marked seminal fluid is transferred and stored in the female mosquitoes' spermathecae upon successful mating, allowing direct measurement of female mating interaction with marked males. Rhodamine $B$ is a thiol-reactive fluorone dye commonly used as a biomarker for ecological and behavioral research studies in animals including insects20. For mosquito studies, RhB is introduced by feeding with sugar or honey water containing dissolved RhB powder $18,19,21,22,23,24$. Upon uptake, the RhB dye binds to proteins, staining body tissue with a reddish-pink stain that fluoresces bright orange under a fluorescent light source.

The strong fluorescence signal and stability of the marking, coupled with its ability to stain insect seminal fluids, allows for the monitoring of the transfer of marked seminal fluid from the labelled male to the sperm storage organs of the female insect for mating studies ${ }^{18,19,21,24}$. The use of $\mathrm{RhB}$ in a male mating competitiveness assay not only allows direct measurement of the mating interaction of females with either marked and unmarked males, but the results can also be obtained within $24 \mathrm{~h}$ as it obviates the process of determining the egg viability, which typically requires about 10 to 14 days. Furthermore, this method overcomes the potential loss of data when the female mosquitoes do not blood-feed or die before ovipositioning. This is particularly crucial because in semi-field trials, where female mosquitoes are prone to damage and death during post-mating collection using a backpack or mechanical aspirator. To address the current limitations of using female fertility to we present an alternative 
method that uses RhB staining to directly measure male mosquito mating competitiveness. The method simplifies the workflow, shortened experimental time from about two weeks to one day, allowing for more experimental replicates to be carried out and allows comparison between two release strains. This protocol will be suitable for laboratories that are embarking on male release-based mosquito population suppression programs, and may be used for routine quality control and strain evaluation.

\section{Protocol}

\section{Rearing of mosquitoes}

1. Conduct all mosquito rearing and the male mating competitiveness assay under standard insectary conditions of $27 \pm 1^{\circ} \mathrm{C}$ and $75-80 \%$ relative humidity, with a photoperiod of $12 \mathrm{~h}: 12 \mathrm{~h}$ light: dark cycles.

2. Designate the two sets of competing males as Set A and Set B for easy reference in the methodology described in this paper. Rear the mosquitoes under standardized conditions to ensure a fair comparison of their fitness during the assay. Rear the mosquitoes at a larval density of 500 larvae in $2 \mathrm{~L}$ of water and feed them with ground fish food powder ad libitum.

NOTE: For the generation of the representative results, Set $A$ and Set $B$ were the inbred and outcross males of the Wolbachia-infected Ae. Aegypti, respectively.

3. Sex male and female mosquitoes at the pupal stage, and contain them separately in cages (see the Table of Materials) of dimensions W $32.5 \mathrm{~cm}$ x D $32.5 \mathrm{~cm}$ x H 32.5 cm, with mesh size of $150 \times 150$ and $160 \mu \mathrm{m}$ aperture. Maintain all adult mosquitoes with $10 \%$ sucrose solution.

\section{Preparation of male and female mosquitoes}

1. Sex the male and female mosquitoes at the pupal stage according to their size differences (male pupae being smaller than female pupae) (Figure 1).

2. For each set of mosquitoes (Set A or Set B), transfer 100 male pupae each into a prelabelled cage for sucrose feeding or RhB-sucrose feeding.

3. Place the female pupae in small batches of $40-50$ per cage. Upon the emergence of the imagoes, check the cages for the presence of male mosquitoes.

NOTE: Adult male mosquitoes are smaller than females and have bushier and hairier antennae (Figure 2). Use only virgin female mosquitoes for the mating competitiveness assays. The use of pre-inseminated females will render all resulting data invalid. Thus, extreme care must be taken during sexing at the pupae stage. Do not use the female mosquitoes from a cage that has been contaminated with male mosquitoes. Extra cages of females should be prepared.

\section{Preparation of $0.2 \% \mathrm{RhB}$ - sucrose solution}

NOTE: RhB is a green powder in dry form and reddishpink in solution. Standard personal protective equipment (PPE: laboratory protection gown, nitrile gloves, and eye protection) shall be worn when handling this chemical. To avoid inhalation, weigh the RhB powder in a fume hood.

1. To prepare a $0.2 \% \mathrm{w} / \mathrm{v}$ RhB-sucrose solution, dissolve $200 \mathrm{mg}$ of RhB powder for every $100 \mathrm{~mL}$ of $10 \% \mathrm{w} / \mathrm{v} /$ sucrose solution. Mix well to ensure all the powder is dissolved.

NOTE: As RhB is light-sensitive, use amber bottles, or wrap clear bottles completely with aluminum foil. 


\section{Feeding of male mosquitoes}

NOTE: Data from RhB-sucrose feeding optimization is presented in Supplemental Material, section 1.

1. Prepare 20 sugar feeder bottles with a wick. Add $10 \mathrm{~mL}$ of $10 \%$ sucrose in 10 feeder bottles and $10 \mathrm{~mL}$ of $0.2 \%$ RhB-sucrose solution in the other 10 feeder bottles (use amber bottles, or wrap the bottles in aluminum foil).

2. Place the feeder bottles in the respective male cages ( 5 bottles per cage) prepared in steps 2.2 and 2.3. Allow the male mosquitoes to feed for three days before the mating experiment.

\section{Checking for RhB fluorescence in male mosquitoes}

1. Aspirate RhB-sucrose-fed male mosquitoes, and observe them under a fluorescence stereo microscope to ensure that all the RhB-sucrose-fed male mosquitoes have been successfully marked with $\mathrm{RhB}$.

2. Turn on the mercury burner lamp and the stereo microscope. Allow the light source of the mercury burner lamp to stabilize for $10 \mathrm{~min}$. Set the fluorescence filters for red fluorescence protein 1 (RFP1) (excitation wavelength $540 \mathrm{~nm}$, emission wavelength $625 \mathrm{~nm}$ ).

3. Aspirate a small number of mosquitoes (four or five) at a time into the glass tube of the oral aspirator. Through the glass tube, observe the body of the male mosquitoes under the fluorescence stereo microscope. Exclude male mosquitoes not marked with $\mathrm{RhB}$ from the experiment. NOTE: The abdomen of male mosquitoes marked with RhB will appear pink under white light (Figure $3 \mathbf{A}$ ) and glow bright orange under fluorescent light (Figure 3B).
4. Transfer Set A male mosquitoes into 12 paper cups secured with netting (mesh size $150 \times 150,160 \mu \mathrm{m}$ aperture); 6 cups each with 10 sucrose-fed male mosquitoes and the other 6 cups each with $10 \mathrm{RhB}$ sucrose-fed male mosquitoes. Repeat this step for Set B male mosquitoes.

\section{Mating competitiveness assay}

1. Set up $12 \mathrm{~W} 60 \mathrm{~cm} \times \mathrm{D} 60 \mathrm{~cm} \times \mathrm{H} 60 \mathrm{~cm}$ cages with mesh size $44 \times 32,650 \mu \mathrm{m}$ aperture for the mating assay. In each of the six cages, include 10 Set A (RhBmarked) male mosquitoes, 10 Set B (unmarked) male mosquitoes, and 10 virgin wild-type female mosquitoes. In the other six cages, include 10 Set A (unmarked) male mosquitoes, 10 Set B (RhB-marked) male mosquitoes, and 10 virgin wild-type female mosquitoes. Label these cages to clearly distinguish between the two mating combinations.

NOTE: Based on experience, a $60 \mathrm{~cm}$ x $60 \mathrm{~cm} \times 60 \mathrm{~cm}$ cage was used for the mating assay as a smaller cage may encourage mixed mating.

2. Place the respective cups of males prepared in step 5.4 into the mating cages according to the label in step 6.1 . Remove the netting and gently tap the cup to jostle the males out of the cup. Carefully remove the paper cup and the netting from the cage to ensure no mosquitoes escape from the cage. Allow the male mosquitoes to acclimate in the mating cage for at least an hour.

3. Using an oral aspirator, transfer virgin wild-type female mosquitoes into 12 paper cups, with each cup containing 10 mosquitoes.

4. After the acclimation period for the male mosquitoes, transfer one cup of females into every mating cage and 
remove the netting. Gently jostle the cup to encourage any remaining female mosquitoes out of the cup. Carefully remove the paper cup and the netting from the cage to ensure no mosquitoes escape from the cage.

5. Allow mating to take place for $3 \mathrm{~h}$.

NOTE: Recommended mating duration was determined through prior observations of wild-type Ae. aegypti. In experiments involving 10 females and 20 males being held in a $60 \mathrm{~cm} \times 60 \mathrm{~cm} \times 60 \mathrm{~cm}$ cage, $90 \%$ female insemination was achieved in $3 \mathrm{~h}$ (Supplemental Material, section 2). Do not disturb the cage during this period as agitation could lead to interrupted and mixed mating. Mixed mating (where the female has mated with both marked and unmarked males) results in a bias towards RhB-marked males as it is difficult to distinguish unmarked sperms from RhB-marked sperms under a fluorescence microscope.

6. To terminate the mating experiment, remove all the mosquitoes from each cage using a mechanical aspirator. Cold anesthetize the mosquitoes on ice for at least $5 \mathrm{~min}$. When the mosquitoes are fully anesthetized, gently pick up the female mosquitoes and house them in a separate paper cup secured with netting (mesh size $150 \times 150,160 \mu \mathrm{m}$ aperture). Label the paper cup by transferring the respective label from the mating cage onto the paper cup.

NOTE: It is possible to pause the experiment at this point and maintain the females with $10 \%$ sucrose solution. The RhB-marked seminal fluid will remain stable inside the female spermathecae for at least a week. It is best to keep the females alive before dissection as dead and desiccated specimens are difficult to dissect.

7. To score the female spermathecae, cold anesthetize the female mosquitoes on ice for at least $5 \mathrm{~min}$ before dissection under a stereo microscope (Video 1). Examine the spermathecae under a compound light microscope (magnification 100x) for their insemination status (Figure 4). For inseminated individuals, determine whether the spermathecae contain RhB-marked seminal fluid by examining them under the fluorescence stereo microscope equipped with an RFP1 filter and a camera imaging system.

NOTE: When using a fluorescence stereo microscope with an attached imaging system, it is recommended to utilize a prolonged exposure time $(5 s)$ to increase the detection sensitivity. If the female mosquito has mated with a marked male, her spermathecae will fluoresce bright orange (Figure 5A). However, if the female mosquito has mated with an unmarked male, her inseminated spermathecae will not fluoresce (Figure $5 B)$.

\section{Disposal of RhB waste}

1. Treat aqueous RhB waste with activated carbon ${ }^{25}$ before discharging it as general waste-water. Dispose of solid RhB waste (mosquitoes marked with RhB, paper towels, and wicks soaked with $\mathrm{RhB}$ ) as chemical waste. Don standard PPE when handling RhB waste.

\section{Representative Results}

wAlbB-SG is a localized (Singapore) Ae. aegypti line stably infected with the wAlbB strain of Wolbachia. Using the protocol described in this paper, we evaluated the male mating competitiveness of an inbred and an outcrossed line of wAlbB-SG to determine if inbreeding results in a loss in male mating fitness. The inbred line had been maintained for 11 generations in the insectary, while the outcrossed line was generated by backcrossing the females with wild-type male 
Ae. aegypti. Males from the inbred and outcrossed lines were competed against each other for mating with wild-type wildtype female Ae. aegypti. The mating competitiveness assay was conducted in triplicate.

The results indicated that $\mathrm{RhB}$ did not affect the fitness of the males as the data for female insemination was not biased towards or against mating with RhB-sucrose-fed males (Table 1 and Figure 6) As RhB does not affect the mating fitness of the males, we proceed to analyse the data based on the percentage of inseminated females mated by either the inbred or outcrossed line (Table 2 and Figure 7). The result across the experimental triplicates were consistent; there was a significantly higher percentage of females mated with the outcross males than with the inbred males in all three replicates $(P \leq 0.05$, Mann-Whitney $U$-test). These results suggest a potential loss in male mating fitness after several generations of inbreeding in the laboratory.

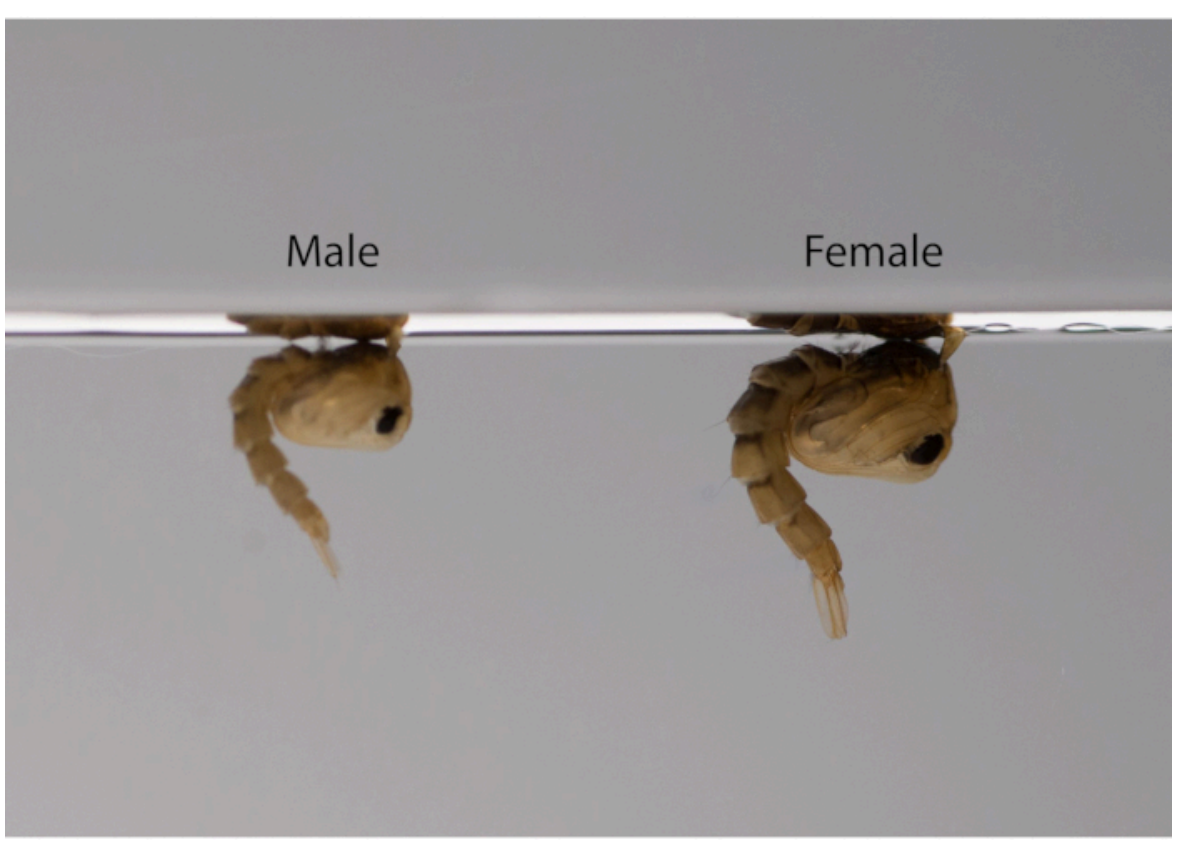

Figure 1: Lateral view of male (left) and female (right) Aedes aegypti pupae. Under the same rearing conditions, Ae. aegypti can be sexed at the pupal stage according to size; males are significantly smaller than females. Please click here to view a larger version of this figure. 
A

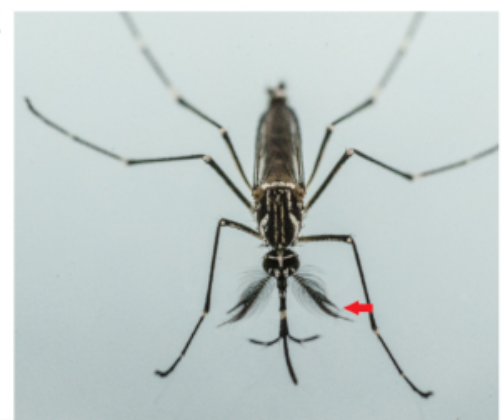

B

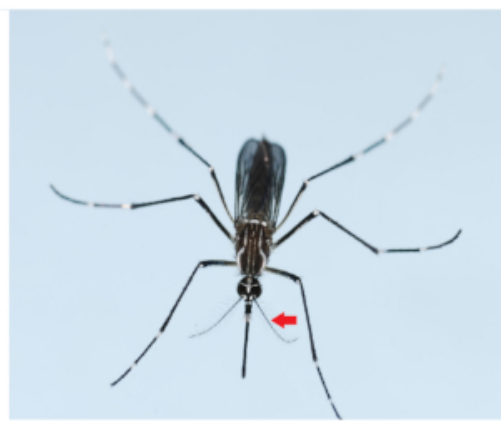

Figure 2: Differentiation of male (left) and female (right) Aedes aegypti adults. Adult male mosquitoes (left) have bushier and hairier antennae than the adult female; the red arrows indicate the antennae. Please click here to view a larger version of this figure.
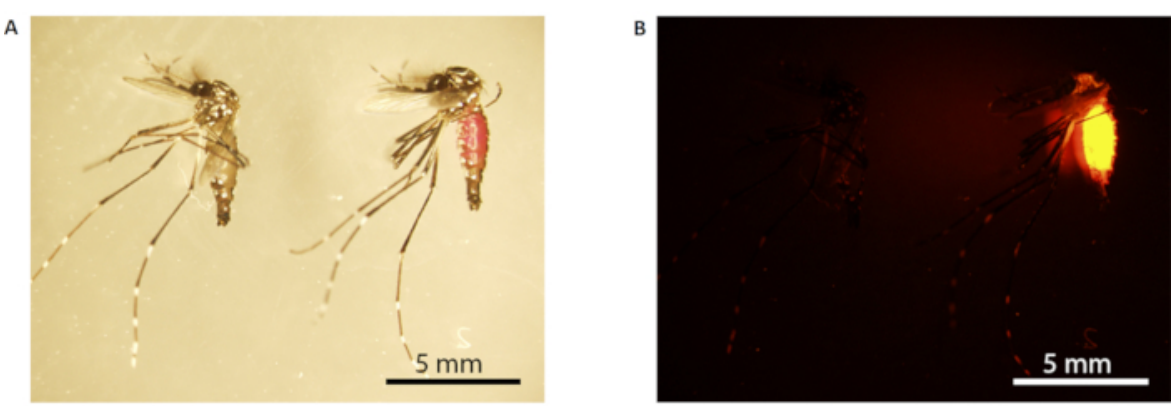

Figure 3: Rhodamine B marking of male mosquito. (A) Light microscopy; (B) fluorescence microscopy. The mosquito on the left is unmarked (fed with $10 \% \mathrm{w} / \mathrm{v}$ sucrose), while the one on the right is marked (fed with $0.2 \%$ RhB-sucrose). Marked mosquitoes have a visible pink abdomen under white light (the mosquito on the right in A), which fluoresces bright orange under fluorescence microscopy (B). Scale bars $=5 \mathrm{~mm}$. Abbreviation: RhB $=$ Rhodamine B. Please click here to view a larger version of this figure. 


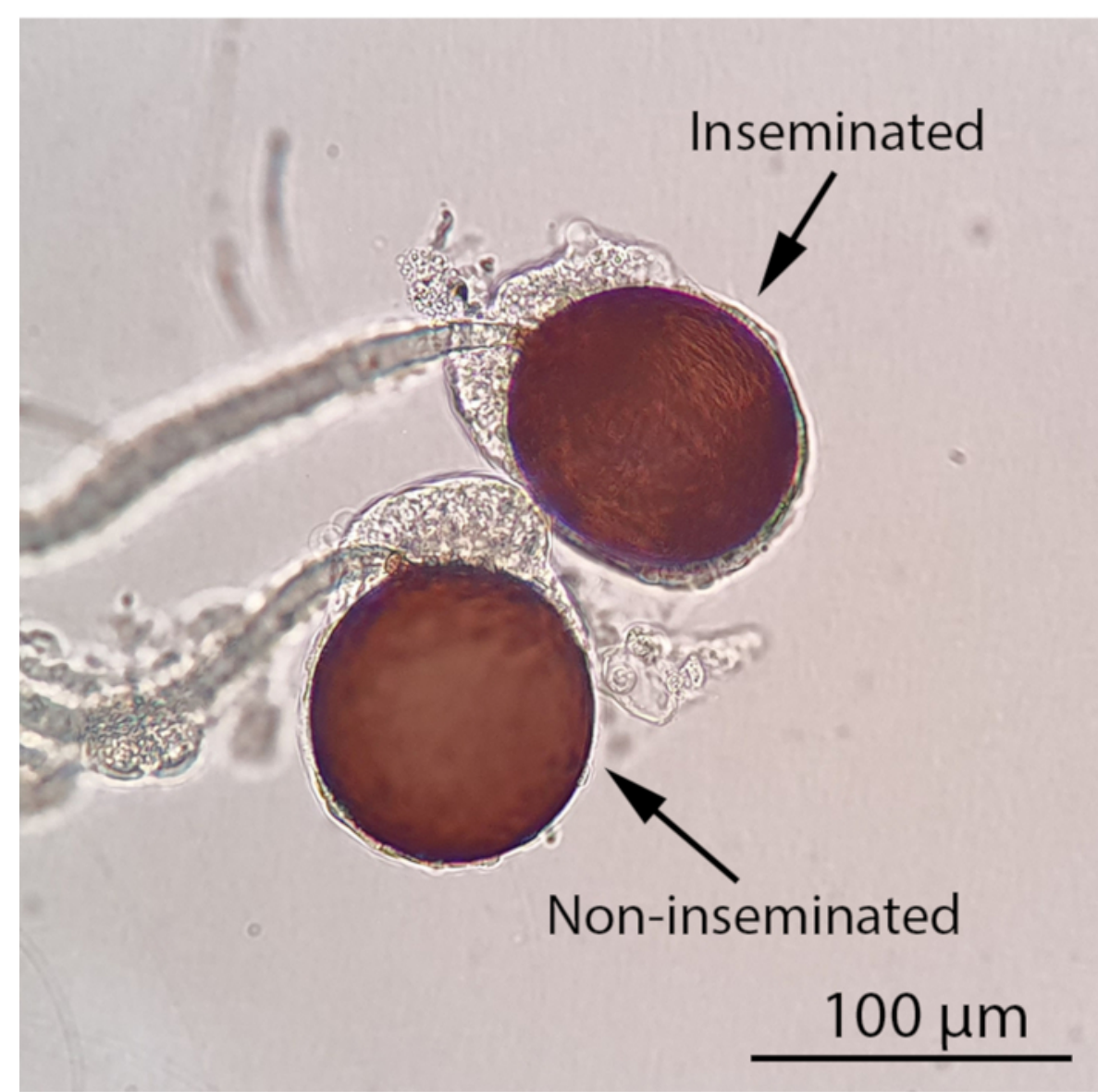

Figure 4: Inseminated and non-inseminated female spermathecae under a compound light microscope (100x magnification). The insemination status of a female mosquito can be determined by observing its spermathecae under a compound light microscope. An inseminated female mosquito will contain at least one filled spermatheca while all three spermathecae of a non-inseminated female mosquito will be empty. Thread-like, motile sperm will be visible in a filled spermatheca under a compound light microscope. Scale bar $=100 \mu \mathrm{m}$. Please click here to view a larger version of this figure. 
A

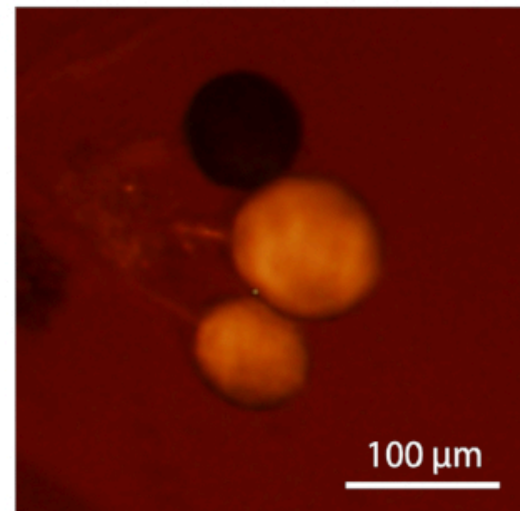

B

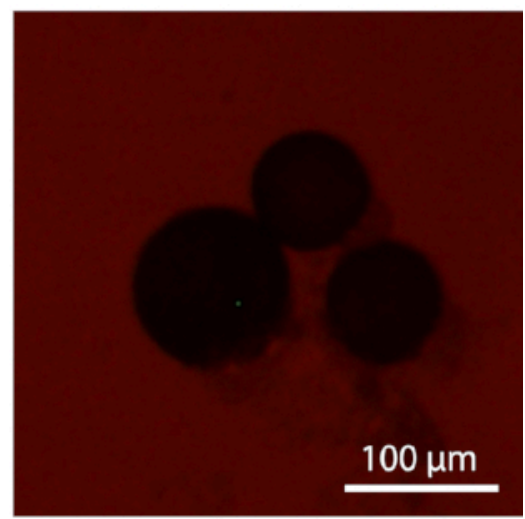

Figure 5: Female mosquito spermathecae inseminated with seminal fluids under a fluorescence stereo microscope. (A) RhB-marked and (B) unmarked spermathecae inseminated with RhB-marked seminal fluids will fluoresce bright orange under fluorescence microscopy. Scale bars $=100 \mu \mathrm{m}$. Please click here to view a larger version of this figure.
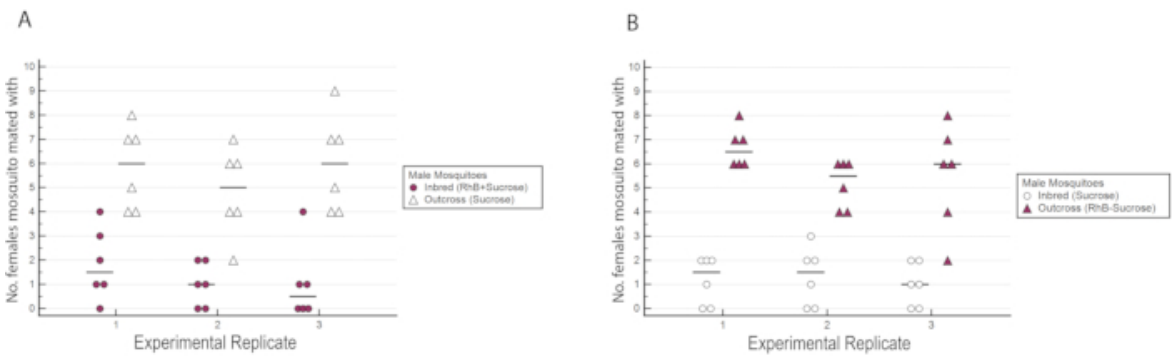

Figure 6: Number of wild-type females inseminated by either the inbred or outcross males in the experimental triplicates with reciprocal marking. (A) The inbred males were marked with RhB while the outcross males were unmarked. (B) The outcross males were marked with RhB while the inbred males were unmarked. A higher number of females was observed to have mated with outcross males regardless of their marking status. Please click here to view a larger version of this figure. 


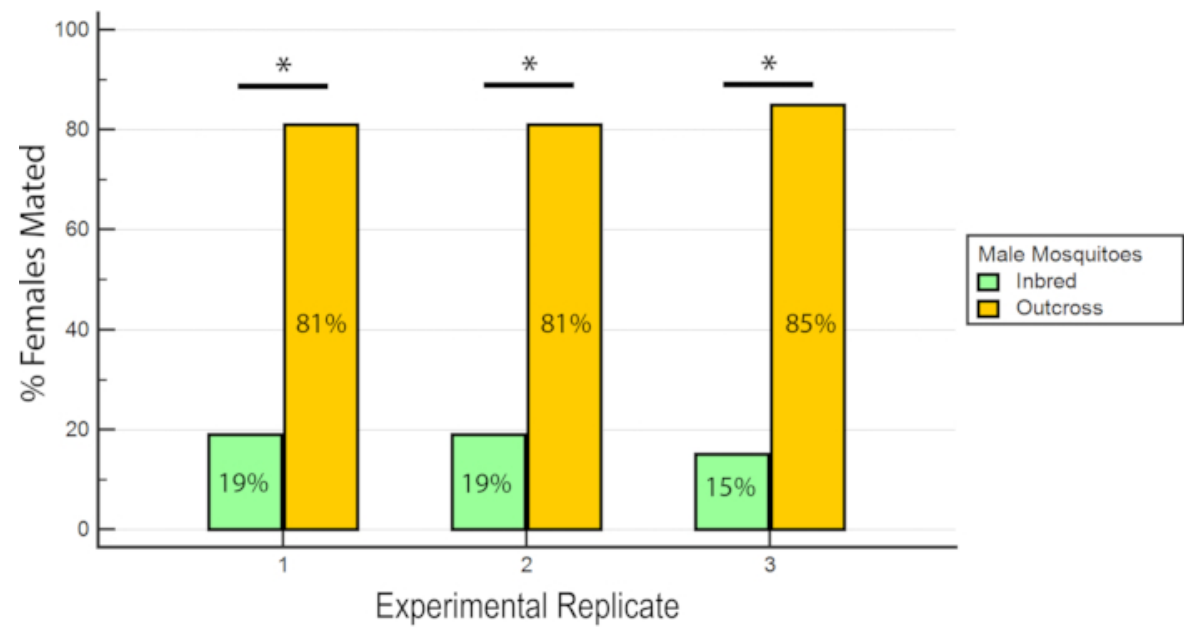

Figure 7: Proportion of inseminated females mated with inbred or outcross males in the 3 experimental replicates. For each experimental replicate, there is a significantly higher percentage of females mated with the outcross males $(P \leq$ 0.05, Mann-Whitney $U$-test). Please click here to view a larger version of this figure.

Video 1: Dissection of female Aedes aegypti for spermathecae under a light stereo microscope. Please click here to download this Video.

\begin{tabular}{|c|c|c|c|c|c|}
\hline & $\begin{array}{l}{ }_{+} \mathrm{x} \text { Inbred } \\
(\mathrm{RhB}) \overbrace{}^{\wedge}\end{array}$ & $\begin{array}{l}\text { }+\mathrm{x} \text { Outcross } \\
\text { (unmarked) } \partial^{\star b}\end{array}$ & $\begin{array}{c}\text { } \mathrm{x} \text { Inbred } \\
\text { (unmarked) } \overbrace{}^{\wedge} \mathrm{C}\end{array}$ & $\begin{array}{c}+x \text { Outcross } \\
(\mathrm{RhB}) \hat{\jmath}\end{array}$ & $\begin{array}{c}\text { Overall } \\
\text { insemination rate } \\
(a+b+c+d / 120)\end{array}$ \\
\hline Replicate 1 & 11 & 35 & 7 & 40 & $77.5 \%(93 / 120)$ \\
\hline Replicate 2 & 6 & 29 & 8 & 31 & $61.7 \%(74 / 120)$ \\
\hline Replicate 3 & 6 & 36 & 6 & 33 & $67.5 \%(81 / 120)$ \\
\hline
\end{tabular}

Table 1: Number of females mated with RhB-marked and unmarked wAlbB-Sg Aedes aegypti inbred and outcross males. A total number of 120 females were used in each replicate. 


\begin{tabular}{|c|c|c|}
\hline & \multicolumn{2}{|c|}{ Percent inseminated females } \\
\hline & Inbred males & Outcross males \\
\hline Replicate 1 & $19 \%(18 / 93)$ & $81 \%(75 / 93)$ \\
\hline Replicate 2 & $19 \%(14 / 74)$ & $81 \%(60 / 74)$ \\
\hline Replicate 3 & $15 \%(12 / 81)$ & $85 \%(69 / 81)$ \\
\hline
\end{tabular}

Table 2: Percentage of inseminated females mated with wAlbB-Sg Aedes aegypti inbred and outcross males.

Supplemental Figure S1: Comparison of workflow for RhB-based and conventional mating competitiveness assay. In comparison to the conventional mating competitiveness assay, the simplified and shortened workflow for RhB-based mating competitiveness assay significantly reduces the experimental duration. Please click here to download this File.

\section{Supplemental Figure S2: Kaplan Meier survival curves of} male adult Aedes aegypti during and after feeding with $0.2 \%$ and $0.4 \%$ rhodamine B-sucrose feeding. Percent survival of (A) male wild-type and (B) wAlbB-Sg Ae. aegypti during and after three days of feeding on $0.2 \%$ and $0.4 \%$ RhBsucrose, compared to controls that were fed with sucrose only. Please click here to download this File.

Supplemental Table S1: Insemination rate of females in a W $60 \mathrm{~cm} \times \mathrm{D} 60 \mathrm{~cm} \times \mathrm{H} 60 \mathrm{~cm}$ cage (ratio of 10 females to 20 males) at 1-, 2-, and 3-h time points. Please click here to download this Table.

\section{Discussion}

Marking is commonly used in entomological research to study insect population dynamics, dispersal, behavior, and mating biology ${ }^{26}$. In SIT and IIT programs, marking is carried out to differentiate the release strain from the field population to study their dispersal and optimize the release ratio. The marking methods used include genetic marking 27,28 , incorporating isotopes in larval food ${ }^{29,30}$, fluorescent dust ${ }^{31}$, and $d y e^{32}$. For the suppression of mosquito populations using SIT or IIT, where male mating fitness is a critical component, fluorescent dyes have been used as markers to study mosquito mating biology ${ }^{18,19}$

Conventionally, assessment of male mating competitiveness of the release strain has been assessed using female fertility assays. However, this assay is time-consuming and laborintensive due to downstream experimental processes postmating (Supplemental Figure S1). These processes include blood-feeding of the females, egg collection, hatching of the eggs, and enumeration of the proportion of hatched eggs to determine egg viability. On average, this assay requires 30 man-hours and two weeks of experimental work (starting from setting up of the competitiveness assay cages) to the final determination of male mating competitiveness.

his paper presents the use of a fluorescent dye, RhB, (fed as $0.2 \% \mathrm{RhB}$-sucrose to the mosquitoes, Supplemental Figure S2) to directly measure mating interactions between females and RhB- marked males. While this protocol requires a fluorescence stereo microscope, it obviates the need to perform the time-consuming experimental procedures 
mentioned above. On average, this RhB-based assay requires approximately 10 man-hours and about a day to obtain data equivalent to that from female fertility assays. This This $>90 \%$ time savings allows researchers researchers to perform multiple experimental replicates, providing a more robust validation of male mating fitness. In addition, this assay can be used to compare the mating competitiveness between two sterile or Wolbachia-infected mosquitoes lines.

This type of comparison is not possible with conventional female fertility assays, as females would yield non-viable eggs upon mating with both such lines. Notwithstanding, any mixed mating in the experiment will result in bias toward the marked population as it is difficult to identify unmarked sperms in female spermathecae that contain seminal fluid from both RhB-marked and unmarked males. A similar conclusion was made in a study assessing mating competitiveness of Anopheles gambiae using $\mathrm{RhB}^{18}$, whereby a greater proportion of females in the mating assay was found to be mated by marked males. As polyandry is more likely to occur in females that had previously engaged in an interrupted mating ${ }^{33}$, the probability of this occurring was reduced in this study by using fewer mosquitoes (20 males to 10 females) in a larger cage volume $\left(0.216 \mathrm{~m}^{3}\right)$ in these experiments.

The results showed no bias toward the RhB-marked population, indicating that mixed mating was limited. In summary, incorporation of RhB to mark males in a mating competitiveness assay is an economical and rapid way of evaluating male mating fitness. This method also allows for the direct comparison of mating competitiveness between males exposed to different doses of irradiation, reared in different rearing regimes, or those infected with different strains of Wolbachia, making it a valuable tool for the evaluation of male mating fitness for any male release-based mosquito population suppression program.

\section{Disclosures}

The authors declare that they have no competing financial interests.

\section{Acknowledgments}

This study was funded by the National Environment Agency (NEA), Singapore. We thank Mr Chew Ming Fai, Deputy Chief Executive Officer (Public Health), NEA, for his approval to publish the study, and A/Prof Ng Lee Ching, Group Director (Environmental Health Institute Group), NEA, for her support in this study. We also thank Dr Shuzhen Sim and Dr Denise Tan for proofreading the manuscript.

\section{References}

1. Achee, N. L. et al. A critical assessment of vector control for dengue prevention. PLoS Neglected Tropical Diseases. 9 (5), e0003655-e0003655 (2015).

2. Carvalho, D. O. et al. Suppression of a field population of Aedes aegypti in Brazil by sustained release of transgenic male mosquitoes. PLoS Neglected Tropical Diseases. 9 (7), e0003864 (2015).

3. Lees, R. S., Gilles, J. R. L., Hendrichs, J., Vreysen, M. J. B., Bourtzis, K. Back to the future: the sterile insect technique against mosquito disease vectors. Current Opinion in Insect Science. 10, 156-162, (2015).

4. Bourtzis, K. et al. Harnessing mosquito-Wolbachia symbiosis for vector and disease control. Acta Tropica. 132, S150-S163 (2014).

5. Zhang, D., Lees, R. S., Xi, Z., Gilles, J. R. L., Bourtzis, K. Combining the sterile insect technique with 
Wolbachia-based approaches: II--A safer approach to Aedes albopictus population suppression programmes, designed to minimize the consequences of inadvertent female release. PloS One. 10 (8), e0135194-e0135194 (2015).

6. Zheng, $X$. et al. Incompatible and sterile insect techniques combined eliminate mosquitoes. Nature. 572 (7767), 56-61 (2019).

7. Balestrino, F. et al. Gamma ray dosimetry and mating capacity studies in the laboratory on Aedes albopictus males. Journal of Medical Entomology. 47 (4), 581-591 (2010).

8. Bellini, R. et al. Mating competitiveness of Aedes albopictus radio-sterilized males in large enclosures exposed to natural conditions. Journal of Medical Entomology. 50 (1), 94-102 (2013).

9. Helinski, M. E., Parker, A. G., Knols, B. G. Radiation biology of mosquitoes. Malaria Journal. 8 Suppl 2 (Suppl 2), S6 (2009).

10. Aldersley, A. et al. Too "sexy" for the field? Paired measures of laboratory and semi-field performance highlight variability in the apparent mating fitness of Aedes aegypti transgenic strains. Parasites \& Vectors. 12 (1), 357-357 (2019).

11. Axford, J. K., Ross, P. A., Yeap, H. L., Callahan, A. G., Hoffmann, A. A. Fitness of wAlbB Wolbachia infection in Aedes aegypti: parameter estimates in an outcrossed background and potential for population invasion. The American Journal of Tropical Medicine and Hygiene. 94 (3), 507-516 (2016).

12. Benedict, M. Q. et al. Colonisation and mass rearing: learning from others. Malaria Journal. 8 (2), S4 (2009).
13. Ross, P. A., Axford, J. K., Richardson, K. M., EndersbyHarshman, N. M., Hoffmann, A. A. Maintaining Aedes aegypti mosquitoes infected with Wolbachia. Journal of Visualized Experiments: JoVE. (126), 56124 (2017).

14. Ross, P. A., Endersby-Harshman, N. M., Hoffmann, A. A. A comprehensive assessment of inbreeding and laboratory adaptation in Aedes aegypti mosquitoes. Evolutionary Applications. 12 (3), 572-586 (2019).

15. Segoli, M., Hoffmann, A. A., Lloyd, J., Omodei, G. J., Ritchie, S. A. The effect of virus-blocking Wolbachia on male competitiveness of the dengue vector mosquito, Aedes aegypti. PLoS Neglected Tropical Diseases. 8 (12), e3294 (2014).

16. Zhang, D., Lees, R. S., Xi, Z., Bourtzis, K., Gilles, J. R. Combining the sterile insect technique with the incompatible insect technique: III-Robust mating competitiveness of irradiated triple Wolbachia-infected Aedes albopictus males under semi-field conditions. PloS One. 11 (3), e0151864 (2016).

17. Fried, M. Determination of sterile-insect competitiveness. Journal of Economic Entomology. 64 (4), 869-872 (1971).

18. Aviles, E. I., Rotenberry, R. D., Collins, C. M., Dotson, E. M., Benedict, M. Q. Fluorescent markers rhodamine B and uranine for Anopheles gambiae adults and matings. Malaria Journal. 19 (1), 236 (2020).

19. Johnson, B. J. et al. Use of rhodamine B to mark the body and seminal fluid of male Aedes aegypti for markrelease-recapture experiments and estimating efficacy of sterile male releases. PLoS Neglected Tropical Diseases. 11 (9), e0005902 (2017). 
20. Fisher, P. Review of using rhodamine $B$ as a marker for wildlife studies. Wildlife Society Bulletin (1973-2006). 27 (2), 318-329 (1999).

21. Blanco, C. A., Perera, O., Ray, J. D., Taliercio, E., Williams, L., III. Incorporation of rhodamine B into male tobacco budworm moths Heliothis virescens to use as a marker for mating studies. Journal of Insect Science. 6, 5 (2006).

22. Mascari, T. M., Foil, L. D. Laboratory evaluation of the efficacy of fluorescent biomarkers for sugar-feeding sand flies (Diptera: Psychodidae). Journal of Medical Entomology. 47 (4), 664-669 (2014).

23. Sarkar, D., Muthukrishnan, S., Sarkar, M. Fluorescent marked mosquito offer a method for tracking and study mosquito behaviour. International Journal of Mosquito Research. 4, 5-9 (2017).

24. South, A., Sota, T., Abe, N., Yuma, M., Lewis, S. M. The production and transfer of spermatophores in three Asian species of Luciola fireflies. Journal of Insect Physiology. 54 (5), 861-866 (2008).

25. ner, O., Geçgel, Ü., Kolancilar, H., Bayrak, Y. Adsorptive removal of rhodamine $B$ with activated carbon obtained from okra wastes. Chemical Engineering Communications. 204 (7), 772-783 (2017).

26. Hagler, J. R., Jackson, C. G. Methods for marking insects: current techniques and future prospects. Annual Review of Entomology. 46, 511-543 (2001).

27. Scolari, F. et al. Fluorescent sperm marking to improve the fight against the pest insect Ceratitis capitata (Wiedemann; Diptera: Tephritidae). New Biotechnology. 25 (1), 76-84 (2008).
28. Ahmed, H. M. M., Hildebrand, L., Wimmer, E. A. Improvement and use of CRISPR/Cas9 to engineer a sperm-marking strain for the invasive fruit pest Drosophila suzukii. BMC Biotechnology. 19 (1), 85 (2019).

29. Botteon, V., Costa, M. L. Z., Kovaleski, A., Martinelli, L. A., Mastrangelo, T. Can stable isotope markers be used to distinguish wild and mass-reared Anastrepha fraterculus flies? PloS One. 13 (12), e0209921 (2018).

30. Hood-Nowotny, R., Mayr, L., Islam, A., Robinson, A., Caceres, C. Routine isotope marking for the Mediterranean fruit fly (Diptera: Tephritidae). Journal of Economic Entomology. 102 (3), 941-947 (2009).

31. Schroeder, W. J., Mitchell, W. C. Marking Tephritidae fruit fly adults in Hawaii for release-recovery studies. Proceedings of the Hawaiian Entomological Society. 23 (3), 437-440 (1981).

32. Akter, H., Taylor, P. W., Crisp, P. Visibility and persistence of fluorescent dyes, and impacts on emergence, quality, and survival of sterile Queensland fruit fly Bactrocera tryoni (Diptera: Tephritidae). Journal of Economic Entomology. 113 (6), 2800-2807 (2020).

33. Oliva, C. F., Damiens, D., Benedict, M. Q. Male reproductive biology of Aedes mosquitoes. Acta Tropica. 132 Suppl, S12-S19 (2014). 